\section{OPEN ACCESS}

Edited by:

Xiaotong Wang,

Ludong University, China

Reviewed by:

Vengatesen Thiyagarajan,

The University of Hong Kong,

Hong Kong

Yongbo Bao,

Zhejiang Wanli University, China

Jian $\mathrm{Hu}$,

Michigan State University,

United States

*Correspondence:

Qianqian Lyu

laqdo@163.com

Weizhi Liu

liuweizhi@ouc.edu.cn

tThese authors have contributed equally to this work.

Specialty section: This article was submitted to

Aquatic Physiology

a section of the journal

Frontiers in Physiology

Received: 09 March 2018 Accepted: 03 May 2018

Published: 23 May 2018

Citation:

Zhang X, Dai X, Wang L, Miao Y, $X u$ P, Liang P, Dong B, Bao Z,

Wang S, Lyu Q and Liu W (2018)

Characterization of an Atypical

Metalloproteinase Inhibitors Like

Protein (Sbp8-1) From Scallop

Byssus. Front. Physiol. 9:597.

doi: 10.3389/fphys.2018.00597

\title{
Characterization of an Atypical Metalloproteinase Inhibitors Like Protein (Sbp8-1) From Scallop Byssus
}

\begin{abstract}
Xiaokang Zhang ${ }^{1+}$, Xiaoting Dai't, Lulu Wang ${ }^{1}$, Yan Miao', Pingping Xu' ${ }^{1}$, Pengyu Liang ${ }^{1}$, Bo Dong ${ }^{1,2}$, Zhenmin Bao ${ }^{1,3}$, Shi Wang ${ }^{1,2}$, Qianqian Lyu ${ }^{1,2 *}$ and Weizhi Liu',2*
\end{abstract}

1 MOE Key Laboratory of Marine Genetics and Breeding, College of Marine Life Sciences, Ocean University of China, Qingdao, China, ${ }^{2}$ Laboratory for Marine Biology and Biotechnology, Qingdao National Laboratory for Marine Science and Technology, Ocean University of China, Qingdao, China, ${ }^{3}$ Laboratory for Marine Fisheries Science and Food Production Processes, Qingdao National Laboratory for Marine Science and Technology, Qingdao, China

Adhesion is a vital physiological process for many marine molluscs, including the mussel and scallop, and therefore it is important to characterize the proteins involved in these adhesives. Although several mussel byssal proteins were identified and characterized, the study for scallop byssal proteins remains scarce. Our previous study identified two foot-specific proteins (Sbp7, Sbp8-1), which were annotated as the tissue inhibitors of metalloproteinases (TIMPs). Evolutionary analysis suggests that the TIMP genes of Chlamys farreri had gone through multiple gene duplications during evolution, and their potential functional roles in foot may have an ancient evolutionary origin. Focusing on the Sbp8-1, the sequence alignment and biochemical analyses suggest that Sbp8-1 is an atypical TIMP. One significant feature is the presence of two extra free Cys residues at its C-terminus, which causes the Sbp8-1 polymerization. Considering the fact that the no inhibitory activity was observed and it is mainly distributed in byssal thread and plaque, we proposed that this atypical Sbp8-1 may play as the cross-linker in scallop byssus. This study facilitates not only the understanding of scallop byssus assembly, also provides the inspiration of water-resistant materials design.

Keywords: scallop, byssus, TIMP, bioadhesion, cross-linker, Cysteine

\section{INTRODUCTION}

It is already well-known that adhesion is one of the most important physiological processes for marine molluscs, which is vital for food procurement, locomotion, defense, breeding and attachment (Gorb, 2008). In the marine environment, many organisms were adversely affected by tides and waves, and in response to this situation many marine molluscs have evolved their ability to live by adhering themselves to other materials (Yang et al., 2013). Like mussels and oysters can secrete adhesives and cement to affix themselves to the substratum (Wilker, 2011). Adhesive locomotion is an important strategy for gastropods, the mucus released from their foot play an important role in locomotion (Iwamoto et al., 2014). The tentacles in Nautilus can produce glue in a specialized glandular structure to pick up food 
or attach to substrate for mating (von Byern et al., 2012). Therefore, adhesion widely exists in marine molluscs indicating this is a crucial physiological event for these organisms. In addition, it is obvious that the marine adhesion material exhibits remarkable adhesion ability with water-resistance properties, providing inspiration for the design of new materials to meet diverse application requirements (Aldred, 2013). Therefore, extensive amounts of efforts were put to characterize the adhesives from sessile organisms such as mussels, barnacles, or tube-dwelling worms (Naldrett and Kaplan, 1997; Taylor and Waite, 1997).

So far, several significant features for marine mollusc adhesives were discovered based on the characterized mollusc adhesives, including the mussels and barnacles. First, Presence of posttranslational modifications (PTM): Several different types of PTM are detected for the studied marine bioadhesive proteins. For example, phosphorylated proteins (Zhao et al., 2005) and hydroxylated DOPA (Wang and Stewart, 2013) were found in the cement of sandcastle worm and $P$. californica respectively. Glycosylation was detected in byssal threads of marine mussels (Sun et al., 2002), and the tube feet disk nectin presented phosphorylation and glycosylation (Toubarro et al., 2016). Second, Metal ions are found to be critical for the marine adhesive materials. Elemental analysis of cured $P$. californica glue revealed relatively large quantities of $\mathrm{Ca}$ and $\mathrm{Mg}$, which are complexed with the peptidyl-phosphates in the heterogeneous sub granules (Wang and Stewart, 2013). And $\mathrm{Fe}^{3+}$-DOPA coordinative complexes were detected in the protective cuticle and bulk adhesive plaque of the byssus (Harrington et al., 2010). Third, so far, the identified marine adhesion proteins are non-conserved. For example, the protein compositions between scallop and mussel are significantly different, although they all belong to bivalves and adopt the byssus to attach to the substratum (Miao et al., 2015). Therefore, it is important to dissect and characterize the individual compositions of scallop byssus, which was important for the understanding its adhesive mechanisms.

Scallops produce and secrete specialized adhesives that work synergistically in water allowing them to attach themselves in marine environments. The secreted adhesive proteins solidified in sea water and shaped into byssus with excellent flexibility and toughness. In previous studies, we discovered 75 proteins from Chlamys farreri byssus based on transcriptomic approach and further identified seven foot-specific scallop byssal protein (Sbp) components based on proteomic approach (Miao et al., 2015). By sequence alignment, two proteins (Sbp7, Sbp8-1) were annotated as the tissue inhibitors of metalloproteinases (TIMPs) family. It is known that TIMPs can regulate important physiological activities by inhibiting metalloproteinases activity, which are proteinases that participate in extracellular matrix (ECM) degradation (Nagase and Woessner, 1999). In addition, it is suggested that TIMPs have other biological functions. For example, TIMPs are involved in erythroid-potentiating (Stetler-Stevenson et al., 1992) and cell growth-promoting (Hayakawa et al., 1992), and it is found that TIMPs can induce apoptosis and stimulate angiogenesis (Qi and AnandApte, 2015). TIMP also might be involved in antibacterial immune in mollusc (Tegillarca granosa) (Wang et al., 2012). Serine proteases and chorionic proteinase inhibitor from Amphibalanus amphitrite was identified, which is predicted to be possibly involved in either regulating the shell formation or functioning in immunity (Zhang et al., 2015). However, the knowledge for the discovered TIMP homologous (Sbp7, Sbp8-1) is insufficient, which precludes the understanding of its physiological function. Moreover, because TIMPs are present as multiple gene copies in the scallop genome, figuring out their evolutionary relationships may promote better understanding of expressional difference between different copies and provide insights into their potential functional divergence. Here our evolutionary analysis suggests that the TIMPs of $C$. farreri had gone through multiple gene duplications during evolution. Focusing on the Sbp8-1, which was mainly distributed in the byssal thread and plaque, detailed sequence alignment suggests that Sbp8-1 is an atypical TIMP with two extra free Cys residues at its C-terminus. More importantly, biochemical analysis in combination with mutagenesis suggests that two extra free Cys residues cause the Sbp8-1 polymerization. Finally considering the fact that the no inhibitory activity was observed, this atypical Sbp8-1 was proposed to function as the crosslinker in scallop byssus. This study will facilitate the biological function understanding of TIMP homologous (Sbp8-1) in byssus.

\section{MATERIALS AND METHODS}

\section{Sample Collection}

The adult Chlamys farreri used in this study were purchased from the market in Qingdao and the scallops were kept in the aquarium with circulating sea water at around $19^{\circ} \mathrm{C}$. Frosted glass was laid on the bottom of the aquarium in order that the scallops can secret adhesives to form byssus. All experiments in this study were repeated at least three times.

\section{Identification and Evolutionary Analysis of TIMP Genes}

Putative TIMP genes in scallops and other molluscs were identified by performing blastp with an $e$-value threshold of $1 \mathrm{e}^{-5}$, using known TIMP genes of human and fly retrieved from NCBI protein database as queries. The conserved domain was identified by using the CDD tool ${ }^{1}$. And only the candidate genes containing NTR domain (Form complexes with metalloproteinases, such as collagenases, and irreversibly inactivate them) were kept for the following analysis. The identified TIMP protein sequences were aligned using clustalw in the MEGA 6.0 software (Tamura et al., 2013) and a phylogenetic tree of TIMP genes was constructed with the program MrBayes (ver3.2.6) (Huelsenbeck et al., 2001). The MCMC model-jumping method was performed to run for $1,000,000$ generations with a sample frequency of 1000 . In total, 1000 trees were produced, of which the first 250 were discarded as burn-in, while the remaining were summarized to get a consensus tree.

\footnotetext{
${ }^{1}$ https://www.ncbi.nlm.nih.gov/Structure/cdd/wrpsb.cgi
} 


\section{Expression Analysis}

The expression levels of TIMP genes were retrieved from the RNA-seq datasets of $C$. farreri that were generated from a wholegenome sequencing project (Li et al., 2017), including eleven adult tissues/organs (adductor muscle, smooth muscle, foot, hepatopancreas, blood, kidney, female gonad, male gonad, gill, eye, and mantle) and three different foot regions (tip region, middle region, and root region). Illumina sequencing reads were mapped to the genome of $C$. farreri by using the STAR software (Dobin et al., 2013). HTSeq-count script (Anders et al., 2015) was used to count the total number of reads matching genic regions. Finally expression value was calculated as RPKM (reads per kilobase per million mapped reads) using the TMM algorithm in EdgeR software (Robinson et al., 2010). The expression level of a TIMP gene was represented by an average RPKM of three biological replicates. A TIMP gene was defined as foot specific if the following conditions were satisfied: foot RPKM $>50$ and foot RPKM/non-foot RPKM > 100 .

\section{Rapid Amplification of cDNA Ends}

In order to obtain the full-length sequences, $5^{\prime}$-RACE and $3^{\prime}$ RACE were carried out, and the primers were designed based on the known nucleic sequence of Sbp8-1(CF9441.24) as shown the primer 1 in Table 1 . The forward primer of $5^{\prime}$-RACE and the backward primer of $3^{\prime}$-RACE are both used the UPM (Universal Primer Mix) from the kit. The $5^{\prime}$-cDNA and $3^{\prime}$-cDNA libraries were constructed using the SMARTer ${ }^{\mathrm{TM}}$ RACE cDNA Amplification Kit (Clontech, CA). The resulting products were then cloned into PMD18-T vector to get the PMD18-T-Sbp8-1 plasmid, which was transformed into E. coli Top10. Finally, the positive clones were confirmed by sequencing.

\section{Plasmid Construction and Recombinant Protein Expression and Purification}

The Sbp8-1 gene with termination codon and cleavage sites of restriction endonucleases ( $\mathrm{Bam} \mathrm{HI}, \mathrm{XhoI}$ ) were obtained by PCR amplification from the plasmid PMD18-T-Sbp8-1 using primer 2 shown in Table 1. The expression vector pET32a was utilized to construct recombinant plasmid, which was transformed into E. coli BL21(DE3) for expression. The cultures were grown in $\mathrm{LB}$ media with $0.1 \mathrm{mg} \cdot \mathrm{ml}^{-1}$ Ampicillin at $37^{\circ} \mathrm{C}$. When the $\mathrm{OD}_{600}$ of the cultures reached $0.6-0.8$, the cells were induced by adding isopropyl- $\beta$-D-thiogalactopyranoside (IPTG) to a final concentration of $0.2 \mathrm{mM}$. Then, the cultures were grown at $16^{\circ} \mathrm{C}$ for $15 \mathrm{~h}$. The cells were harvested by centrifuging $3,000 \times g$ for $30 \mathrm{~min}$ at $4^{\circ} \mathrm{C}$ and the cell pellets were resuspended in $20 \mathrm{~mL}$ ice-cold binding buffer $(20 \mathrm{mM}$ Tris- $\mathrm{HCl}, 20 \mathrm{mM}$ imidazole,
$500 \mathrm{mM} \mathrm{NaCl}, \mathrm{pH}$ 8.5). Cells were lysed by using a sonicator with $5 \mathrm{~s}$ pause every $10 \mathrm{~s}$ at $40 \%$ power for $25 \mathrm{~min}$. The resulting sample was subsequently centrifuged $\left(12,000 \times g, 25 \mathrm{~min}, 4^{\circ} \mathrm{C}\right)$ to remove insoluble fractions.

The final supernatant was applied to pre-equilibrated $\mathrm{Ni}$ NTA matrix and shaking gently at $4^{\circ} \mathrm{C}$ for $1 \mathrm{~h}$. After washing with the binding buffer, Sbp8-1 protein was eluted using elution buffer containing $500 \mathrm{mM}$ imidazole in binding buffer. The corresponding fractions were concentrated to $10 \mathrm{mg} \cdot \mathrm{ml}^{-1}$ for further analysis. The resulting protein was loaded on HiPrep Sephacryl S-200 HR (GE) to monitor the protein polymerization, which was pre-equilibrated with the binding buffer without imidazole. The flow rate is $0.5 \mathrm{ml} \cdot \mathrm{min}^{-1}$ and the peak fraction was collected and detected by SDS-PAGE. For the polyclonal antibody preparation, the fusion Trx tag of recombinant Sbp8-1 was removed by protease (Supplementary Figure 1).

\section{Site-Directed Mutagenesis}

QuikChange Site-Directed Mutagenesis Kit (Agilent technology, Santa Clara, CA, United States) was used to perform the sitedirected mutagenesis. The primers M-C164S and M-C186S (Table 1) were used for the mutation of Sbp8-1 ${ }^{\mathrm{C} 164 \mathrm{~S}, \mathrm{C} 186 \mathrm{~S}}$. The expression and purification method of the mutant protein is the same as that of Sbp8-1.

\section{Localization of Sbp8-1 in Chlamys farreri Byssus Based on Western Blot Scallop Byssal Protein Extraction}

Protein extraction was carried out according to the previous experimental method (Miao et al., 2015). The collected byssus from Chlamys farreri were rinsed several times with deionized water and cut into different parts as the picture showed (Figure 3A). Briefly, the corresponding fractions were grinded into powder in liquid nitrogen and incubated with extraction buffer ( $5 \%$ acetic $(v / v), 6 \mathrm{M} \mathrm{GdnHCl,} 2 \mathrm{mM}$ EDTA, $10 \mathrm{mM}$ DTT) at $37^{\circ} \mathrm{C}$ for $1 \mathrm{~h}$. After centrifugation $\left(12,000 \times g, 25 \mathrm{~min}, 4^{\circ} \mathrm{C}\right)$, supernatant was collected and dialyzed against $1 \%$ acetic acid $(v / v)$ at $4^{\circ} \mathrm{C}$. Finally, the extracted protein was dialyzed against deionized water and lyophilized.

\section{Preparation of Polyclonal Antibody}

The recombinant Sbp8-1 protein was excised from SDS-PAGE gel (Supplementary Figure 1) and ground thoroughly as the antigen. The samples were prepared using a typical procedure (Plumari et al., 2010). After four times immunization, the rabbits were sacrificed and serum was collected for antibody purification (ABclonal Biotechnology, Wuhan, China).

TABLE 1 | Primers used in this study.

\begin{tabular}{|c|c|c|}
\hline Primer name & Forward $\left(5^{\prime}-3^{\prime}\right)$ & Backward $\left(5^{\prime}-3^{\prime}\right)$ \\
\hline Primer 1 & CAAGCGATATTGTCATCATTGGGAAAA & AGTGATGCCACAAACACCTTGGGAGT \\
\hline Primer 2 & CGGGATCCTGTACATGTAATCCATACCCAG & CCGCTCGAGTCAACAATCGTTGTCGGTC \\
\hline M-C164S & CAGTAATTGAGTCTITCAACAAAGAACGTTGCGTACCC & CGCAACGTTCTITGTTGAAAGACTCAATTACTGTTGAATTATCG \\
\hline M-C186S & GTGGACCGACAACGATTCTTGACTCGAGCACCAC & GTGGTGCTCGAGTCAAGAATCGTTGTCGGTCCAC \\
\hline
\end{tabular}


Western blot: Western blot analysis of the byssus protein extractions was performed. After electrophoresis, the proteins were electrotransferred to a PVDF membrane for $45 \mathrm{~min}$ at $100 \mathrm{~V}$, and the membrane was blocked by incubating the membrane with $5 \%$ non-fat milk in Tris-buffered saline containing $0.1 \%$ Tween-20 (TBST) for $2 \mathrm{~h}$. The membrane was then incubated overnight at $4^{\circ} \mathrm{C}$ with rabbit polyclonal anti-Sbp8-1 (1:500 dilution) in blocking solution. After rinsing with TBST, the membrane was incubated with diluted (1:5000) peroxidase-conjugated goat anti-rabbit IgG antibody (BBI, New York, United States) for $1 \mathrm{~h}$. Finally, the membrane blots were developed using an enhanced chemiluminescence regent kit (BOSTER Biological Technology, Wuhan, China).

\section{Peptide Mass Fingerprinting Derived From Gel Bands}

The corresponding band was cut with a clean blade and subjected to trypsin digestion after destaining. After washing with $100 \mathrm{mM}$ $\mathrm{NH}_{4} \mathrm{HCO}_{3}$, the gel pieces were rehydrated in $100 \mathrm{mM} \mathrm{NH}_{4} \mathrm{HCO}_{3}$ with 10 ng sequencing grade-modified trypsin (Promega, WI) at $37^{\circ} \mathrm{C}$ overnight. After digestion, the protein peptides were collected, and the gels were washed with $0.1 \%$ TFA in $60 \% \mathrm{ACN}$ to collect the remaining peptides (Shevchenko et al., 2006). Then the samples were analyzed using Easy-nLC nano-flow HPLC system connected to Orbitrap Elite mass spectrometer (Thermo Fisher Scientific, CA, United States). Then the raw files were analyzed using the Proteome Discoverer 1.4 software (Thermo Fisher Scientific, CA, United States). The deduced Sbp8-1 sequence was used as the search template.

\section{Detection of Metalloproteinase Inhibitory Activity}

Succinylated gelatin and type IV collagenase (Sigma-Aldrich) were used for the metalloproteinase inhibitor activity test. The gelatin was succinylated using the method described previously (Rao et al., 1997). And the inhibition assay was carried out in the reaction system including $0.14 \mu \mathrm{M}$ type IV collagenase and $240 \mu \mathrm{g}$ succinylated gelatin in the presence or absent of Sbp8-1, the total volume was adjusted to $150 \mu \mathrm{L}$ using $50 \mathrm{mM}$ borate buffer. The reaction buffer was incubated at $37^{\circ} \mathrm{C}$ for $30 \mathrm{~min}$, then reacted with $0.03 \%$ TNBSA and read at $450 \mathrm{~nm}$ after incubation at room temperature for $20 \mathrm{~min}$ and the relative enzyme activity was monitored.

\section{Circular Dichroism Spectrum Detection}

In order to verify whether the Sbp8-1 protein was folded correctly, the secondary structure of Sbp8-1 was detected by circular dichroism spectrum. The Sbp8-1 protein was digested with protease to remove the fusion tag. After purification, the resulting Sbp8-1 without Trx was dialyzed against phosphate buffer $\left(0.01 \mathrm{M}, \mathrm{pH} 8.0\right.$ with $\left.250 \mathrm{mM} \mathrm{Na}_{2} \mathrm{SO}_{4}\right)$. The protein sample was injected into the quartz cell and then measured within the wavelengths ranging from 190 to $250 \mathrm{~nm}$ (detection step of $1 \mathrm{~nm}$ and a detection time interval of $0.5 \mathrm{~s}$ ). During the test, the phosphate buffer was used as a control and the experiment was repeated three times.

\section{Amino Acid Composition, Sequence Alignment and Structural Modeling}

The TIMP-2 protein sequences with the highest structural similarity to the Sbp8-1 protein were selected for amino acid sequence alignment. The alignment was generated with ClustalW (Figure 4). The molecular weights and theoretical isoelectric points were analyzed on the Expasy server ${ }^{2}$. Signal peptide was predicted with SignalP 4.1 server $^{3}$.

\section{RESULTS AND DISCUSSIONS}

\section{Evolutionary Analysis of TIMP Genes}

To gain insights into the evolution of TIMP gene and its expression in different tissues of scallops, we conducted genomewide search and identified thirteen TIMP genes in the C. farreri genome. In addition, thirteen TIMP genes were identified in scallop Patinopecten yessoensis and oyster Crassostrea gigas respectively, but none in other molluscs (Lottia gigantea, Octopus bimaculoides, and Aplysia californica). The number of TIMPs in bivalves is remarkably higher than those in other molluscs and non-molluscs (e.g., four TIMPs in human and one in fly) suggesting that TIMP gene family in bivalves had expanded notably during evolution.

Our previous study identified seven byssal proteins that were foot specific (Miao et al., 2015). Among them, two TIMPs (Sbp7, Sbp8-1) were discovered through our sequence alignment analysis. Phylogenetic analysis (Figure 1A) indicated the high diversity and deep evolutionary divergence of TIMPs in the bivalve lineage. Many clades contained TIMP genes from both scallops and oyster, implying that the duplication events of TIMP genes even occurred before the divergence of scallop and oyster lineages ( $\sim 25$ million years ago) (Wang et al., 2017). The expression analysis of TIMPs in C. farreri (Figure 1B) revealed a substantial number of foot-specific TIMPs including Sbp7 and Sbp8-1, with remarkably higher expression levels in foot (RPKM: 70-9,000) than in other tissues (0.3-92.0; Figure 1B). These foot-specific TIMPs showed the dispersed distribution across the phylogenetic tree, suggesting that their potential functional roles in foot may have an ancient evolutionary origin. Analysis of the expression level of Sbp8-1 (Cf9441.24) suggests that it is mainly secreted by the middle region and tip region of scallop foot (Figure 2). According to previous reports about the distribution of glands in the scallop foot (Gruffydd, 1978), Sbp8-1 protein may be secreted by the second type of mucous cell which is found all along the ventral surface of the middle region and tip region of the foot. Previous studies showed that different types of proteinase inhibitors were identified in the Zebra mussel (Xu and Faisal, 2008) and Mytilus coruscus byssus (Qin et al., 2016). Also, metalloproteinase inhibitor was identified in the soluble fraction from proximal thread (Qin et al., 2016). Collectively, these findings suggest that TIMP genes may play important roles for the assembly and function of byssus in bivalves, which is likely of ancient evolutionary origin.

\footnotetext{
${ }^{2}$ https://web.expasy.org/protparam/

${ }^{3}$ http://www.cbs.dtu.dk/services/SignalP/
} 

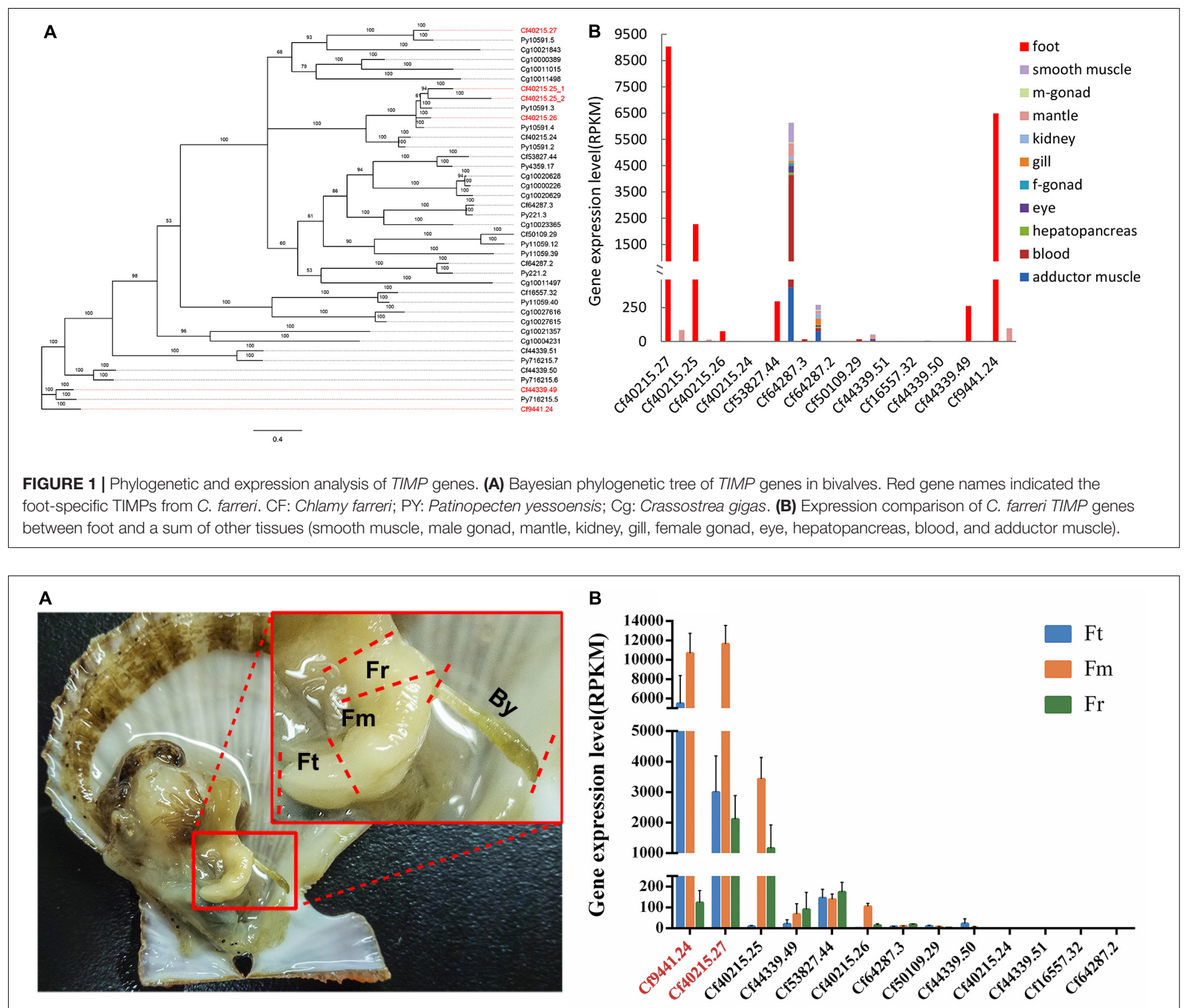

FIGURE 2 | Anatomical structure of Chlamys farreri foot and expression analysis of all of TIMP genes. (A) Anatomy of C. farreri and magnified display of C. farreri foot. It demonstrates the foot contains three different regions as labeled: Fr, root region of foot; Fm, middle region of foot; Ft, tip region foot; By, C. farreri byssus. (B) Expression levels of C. farreri TIMP genes across different foot regions. The genes labeled in red font encode Sbp8-1 (Cf9441.24) and Sbp7 (Cf40215.27) respectively, the standard errors were based on three biological replicates. This analysis indicates that the genes encoding Sbp8-1 and Sbp7 proteins were highly expressed in the middle region and tip region of $C$. farreri foot.

\section{Distribution of Sbp8-1 in C. farreri Byssus}

Detailed visualization demonstrates that the scallop byssus could be divided into four parts (Figure $\mathbf{3 A}$ ) and the protein was extracted for these individual parts. The SDS-PAGE showed that similar protein compositions were observed for the byssal thread and plaque region (Figure 3B). They both contain four major bands with similar $\mathrm{Mw}$ ( 2 bands with $\mathrm{Mw}$ around $40 \mathrm{kDa}$ and 2 bands with $\mathrm{Mw}$ around $20 \mathrm{kDa}$ ). And only one major band was observed with the $\mathrm{Mw}$ around $40 \mathrm{kDa}$ for the sheathed region. However, the two major bands ( 1 band with $\mathrm{Mw}$ around $45 \mathrm{kDa}$ and the other one with $\mathrm{Mw}>75 \mathrm{kDa}$ ) were observed for the root region. Similar to our previous observation, the overall scallop byssal thread protein was composed by three major fractions based on the Mw distribution (Miao et al., 2015). In sum, these observations indicate that protein composition vary in different parts of byssus.

To accurately map the distribution of individual compositions in byssus, the western-blot was carried out. Thereby, we first attempted to over-express these two proteins (Sbp7, Sbp8-1) to obtain as the antigen. However, poor expression level of Sbp7 precluded further investigation of this protein, and then the Sbp8-1 was chosen for the following assay. As shown in 

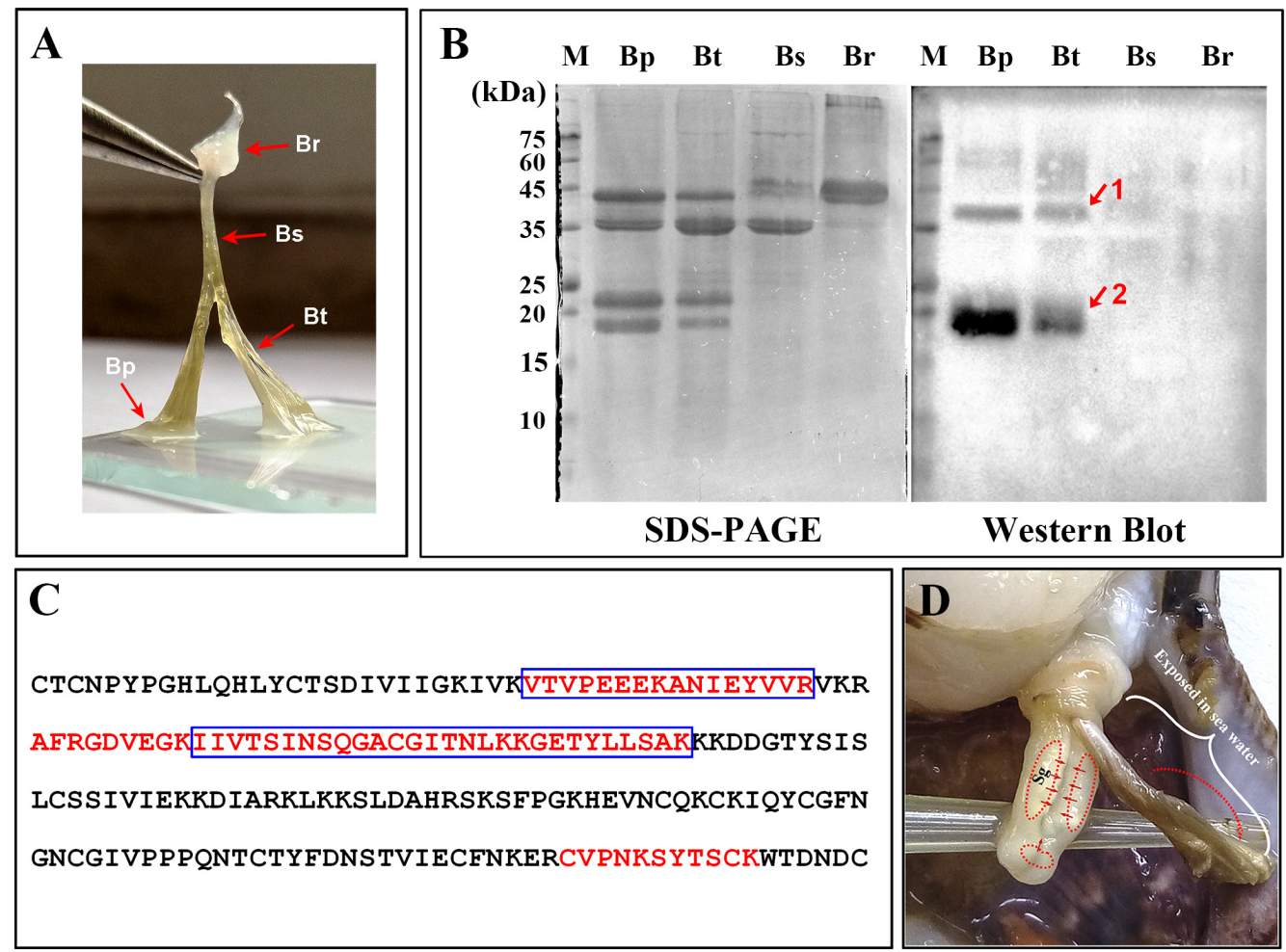

FIGURE 3 | Distribution of Sbp8-1 in Chlamys farreri byssus based on western blot. (A) Picture of $C$. farreri byssus showing it contains four regions; Br, byssal root region; Bs, byssal sheathed region; Bt, byssal thread region; Bp: byssal plaque region. (B) SDS-PAGE and western blot analysis of extracted byssal proteins. The red arrows and numbers indicate the corresponding bands for the further mass spectrometry analysis. (C) The identified unique peptide from the corresponding protein bands in (B). The amino acids in the blue box are the peptides identified in Band 1 and the amino acids in the red font are the peptides identified in Band 2. (D) The secretion and distribution areas of Sbp8-1. Sg, secretory glands. The red solid arrow indicates the secretion areas of the Sbp8-1 in scallop foot based on the expression level analysis. The red dashed arrow indicates the distribution area of Sbp8-1 in scallop byssus based on the western blot analysis.

Figure 3B, the western-blot assay using the anti-Sbp8-1 antibody showed Sbp8-1 was discovered mainly in the byssal thread and plaque region, not in the others. However, two bands were observed for both lanes, one band (band 1) is around the $20 \mathrm{kDa}$, which is close to the Mw of Sbp8-1. Whereas the other band (band 2) is around the $40 \mathrm{kDa}$, which is one fold higher than the $\mathrm{Mw}$ of Sbp8-1. To further validate presence of Sbp8-1 in these two bands, the mass-spectroscopy was carried out. And the result demonstrates that 6 unique peptides were discovered for band 2 and 8 unique peptides for band 1 (Figure 3C). This further confirms that the Sbp8-1 was discovered mainly in the byssal thread and plaque region.

Taken together, it was found that the Sbp8-1 was mainly secreted in the scallop foot middle and tip region based on the expression pattern analysis (Figure 2), and finally distributed in the byssal thread and plaque region as depicted in Figure 3D. However, the transport pathway needs more investigation.

\section{Amino Acid Composition and Sequence Alignment Analysis Amino Acid Composition Analysis}

The amino acid analysis for Sbp8-1, Sbp7 was summarized in Figures 4C,D. One significant feature for Sbp8-1 and Sbp7 compared to others structure-determined TIMPs is the relative high Lys content, which leads to their basic theoretical pI as shown in Figure 4C.

\section{Sequence Alignment}

Sequence alignment was performed as shown in Figure 4A for the two TIMPs from scallop byssal protein (Sbp8-1, Sbp7) with three structure-determined TIMPs (PDB:1BR9, human TIMP2; PDB: 1BQQ, bovine TIMP-2; PDB:3CKI, human TIMP-3). First, it showed the low sequence similarity between the scallop byssal protein (Sbp8-1, Sbp7) and the other TIMPs (Sequence identity $<30 \%$ ); Furthermore, it was found that the C-terminal region is more divergent, and this might be functional relevant because the C-terminal of TIMP may enhance the inhibitory selectivity and binding efficiencies (Willenbrock et al., 1993). TIMP-2 binds to the hemopexin domain of proMMP-2 to form a tight complex through its C-terminal domain (Visse and Nagase, 2003). More surprising, two additional Cys residues are discovered (blue box in Figure 4A). Generally, the TIMPs have 6 disulfides formed by 12 conserved Cys residues (red box in Figure 4A). Previous study demonstrated that insertion of Cys residue in C-terminal of human TIMP-3 results in the human Sorsby's fundus dystrophy (SFD) disease. And the plausible molecular mechanism is the Cys insertion leads to 
A

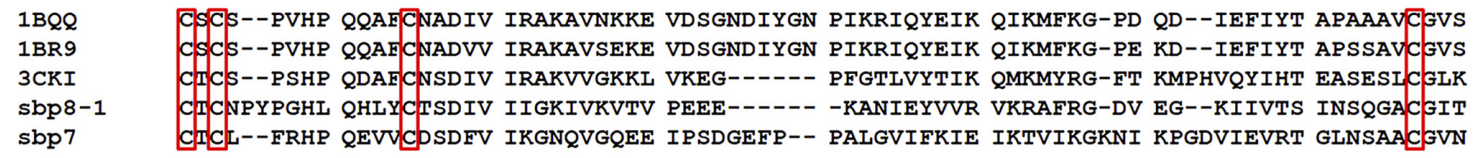

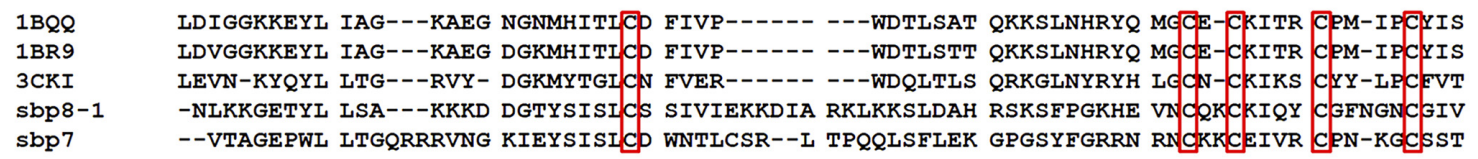

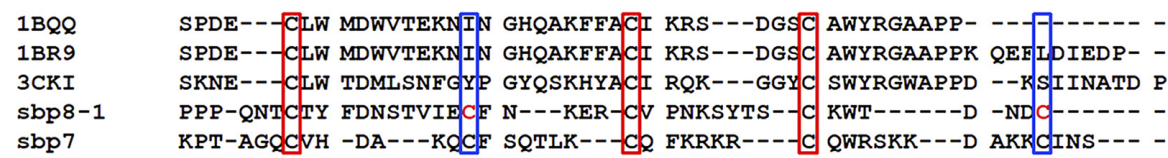

B

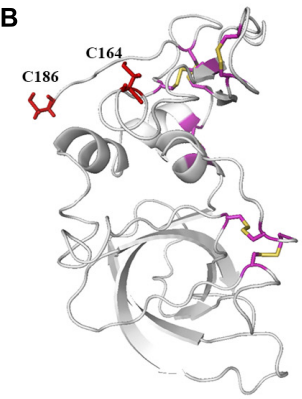

C

\begin{tabular}{lll}
\hline Sequence name & Sbp8-1 & Sbp7 \\
\hline Signal peptide & MKLFVAVLVLCG & MLRASILVLASV \\
Number of amino acid & VQVVFS & VLSFIPDSDG \\
Molecular weight & 20755.9 & 193 \\
Isoelectric point (pI) & 8.87 & 21557.98 \\
\hline
\end{tabular}

D

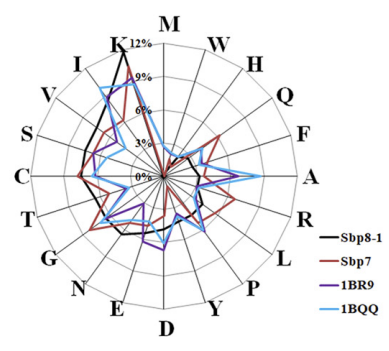

FIGURE 4 | Sequence Characteristics and the basic information of Sbp8-1 and Sbp7. (A) Multiple sequence alignments of the Sbp8-1 and Sbp7 with other structure determined TIMPs. Three others structure-determined TIMPs were chosen due to the relative high sequence similarity to either Sbp8-1 or Sbp7. They include the PDB: 1BR9, human TIMP-2; PDB: 1BQQ, bovine TIMP-2; PDB: 3CKI: human TIMP-3). Red boxes indicate the 12 conserved Cys residues and the blue boxes indicate two extra residues in the C-terminal of Sbp8-1. (B) The three-dimensional structural model of Sbp8-1 generated by I-Tasser (Yang et al., 2015) suggests that two extra Cys present in Sbp8-1 C-terminal do not form disulfide bonds. (C,D) Amino acid composition analysis of Sbp8-1 and Sbp7.

the formation of intermolecular disulfide bridges that cause protein polymerization (Stohr and Anand-Apte, 2012). Also, the structural model of Sbp8-1 generated using the I-Tasser server (Yang et al., 2015) suggests that two extra Cys residues (C164, C186) in Sbp8-1 C-terminal exist as free state (Figure 4B). In sum, the above analyses indicate the Sbp8-1 is an atypical TIMP, which might have distinct biochemical features and unique function in scallop byssus.

\section{Biochemical Characterization of Recombinant Sbp8-1}

Biochemical characterization demonstrates that Sbp8-1 is an atypical TIMP, which may function as the cross-linker in the scallop byssus as mentioned below.

\section{Polymerization}

The recombinant Sbp8-1 (with Trx fusion tag) was loaded on gel filtration chromatography using Hi-Prep Sephacryl S-200 $\mathrm{HR}$ (GE) to monitor the polymerization state. As expected, the Sbp8-1 was eluted in void volume (around $36 \mathrm{~mL}$ ), indicating the polymerization occurs (Figure 5). To explore the relationship between the two additional Cys residues (Figure 3A) and the polymerization, two following assays were carried out. First, the DTT (10 mM) was added in the elution buffer and it turned out that the elution volume shifted to $60 \mathrm{~mL}$, this suggests the presence of intermolecular disulfides might cause the polymerization, which may formed by the two C-terminal extra Cys residues. To confirm this hypothesis, the C164S and C186S double mutation was then generated. The mutant Sbp8$1^{\mathrm{C} 164 \mathrm{~S}, \mathrm{C} 186 \mathrm{~S}}$ was reloaded on the gel filtration chromatography. The elution volume of Sbp8-1 C164S,C186S shifted to the $62 \mathrm{~mL}$ as shown in Figure 5. Our analyses strongly indicate that Sbp8-1 is in the polymerization state, and this polymerization is caused by the two extra free Cys residues in the C-terminal region. It is known that the free thiol groups are typically important for the marine adhesives. For example, in mussel footprint protein mfp- 6 with 11 mol\% cysteine content, and most of them occur as free thiol (Lee et al., 2011). It is known that DOPA exists in the scallop byssal thread and plaque region (Li et al., 2017), it is therefore reasonable to propose that Sbp8-1 may function as the cross-linker through the interaction between free cysteine and DOPA.

\section{Enzymatic Inhibitory Assay}

The biological function annotation based on seqeunce alignment showed that the Sbp8-1 belongs to TIMP-2. Previous study showed that TIMP-2 can inhibit the activity of MMP-2 (type IV 


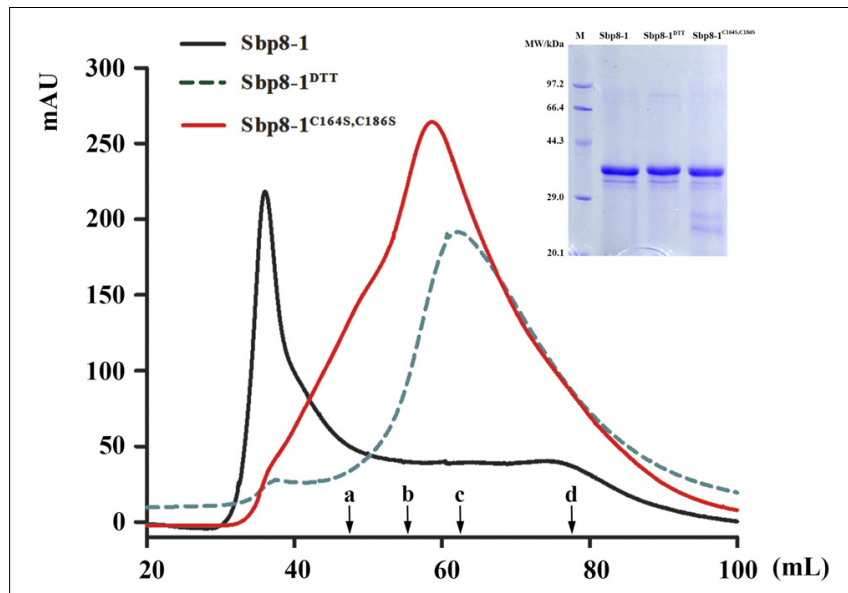

FIGURE 5 | Protein polymerization analysis based on gel filtration. The Sephacryl S-200 High Resolution column was used to evaluate the polymerization state. The solid black line represented the gel-filtration profile of Sbp8-1 running in the binding buffer and the dashed black line represented gel filtration profile of Sbp8-1 running with binding buffer with 10 mM DTT, while the solid red line represented the gel-filtration profile of

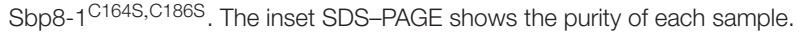
The black arrows indicate the elution volume at the peak of different molecular weight proteins. a, IgG (160 kDa); b, BSA (67 kDa); c, $\beta$-lactoglobulin (35 kDa); d, cytochrome C (12.4 kDa)

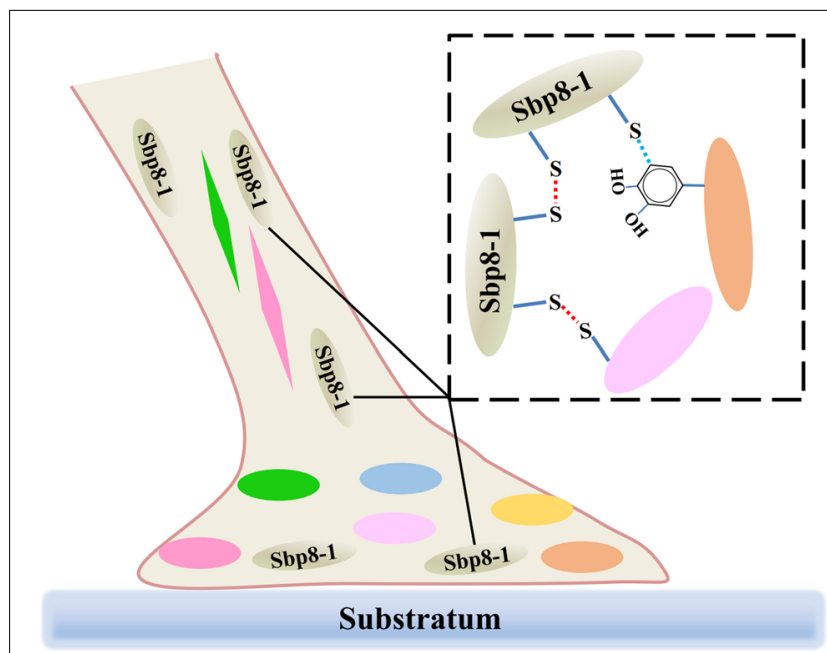

FIGURE 6 | Schematic illustration of the potential cross-linking role of Sbp8-1 protein in the scallop byssus. The left side is a schematic diagram of the structure of the byssus, in which different colors of ovals and diamonds represent different byssal protein components; The dotted box on the right shows the potential cross-linking between Sbp8-1 protein and other proteins.

collagenase) (Tuuttila et al., 1998). In order to test whether Sbp8-1 protein has inhibitory activity toward type IV collagenase, the succinylated gelatin method was used for the assay. However, it turned out that addition of either Sbp8-1 or the fusion tag control (Trx protein) will enhance the type IV collagenase activity (Supplementary Figure 2), suggests that the Sbp8-1 does not have the inhibitory activity toward type IV collagenase, which is different to the canonical human TIMP-2. To verify the folding of Sbp8-1 protein, the circular dichroism was carried out. The result (Supplementary Figure 3) showed that Sbp8-1 protein has obvious secondary structure. In summary, Sbp8-1 protein is an atypical TIMP without metalloproteinase inhibitor activity.

\section{CONCLUSION}

It is known that both the foot and byssus are crucial for scallop, and the exploration of the scallop byssus composition is the first key step to understand its biological function. Focusing on the TIMPs derived from scallop byssus, a comprehensive characterization of Sbp 8-1 was carried out for the first time. Evolutionary analysis suggests that the TIMPs genes of Chlamys farreri had gone through multiple gene duplications during evolution, and their potential functional roles in foot may have an ancient evolutionary origin. Focusing on the Sbp8-1, one of the discovered TIMPs from scallop byssus, sequence alignment and enzymatic inhibitory assay suggest that it is an atypical TIMPs. One significant feature is two extra free Cys residues in C-terminal of Sbp8-1. These two residues might form the intermolecular disulfides resulting in the sbp8-1 polymerization; considering its distribution, we proposed that the Sbp8-1 protein may play the cross-linking role in scallop byssus through the C-terminal extra Cys residues as shown in Figure 6.

\section{AUTHOR CONTRIBUTIONS}

WL, QL, SW, and XZ designed and performed the study and drafted the manuscript. SW, ZB, and LW were in charge of reviewing the data analysis during the manuscript submission process. YM, PX, XZ, and PL contributed experiment materials and analysis tools. XD, XZ, and PX collected the information and analyzed the data. BD and SW participated in study development.

\section{FUNDING}

This work was supported by National Natural Science Foundation of China (31472258, U1706203); Key Research and Development Plan of Shandong Province (2016GSF115008), and the Fundamental Research Funds for the Central Universities (201822024, 201841001, and 201762001).

\section{ACKNOWLEDGMENTS}

We are very grateful to Dr. Xiaoju Dou for the help of using laser scanning confocal microscope. The authors gratefully acknowledge Shanghai Omicsspace Biotechnology Co., Ltd., for technical assistance with the peptide/protein identification.

\section{SUPPLEMENTARY MATERIAL}

The Supplementary Material for this article can be found online at: $\quad$ https://www.frontiersin.org/articles/10.3389/fphys.2018. 00597/full\#supplementary-material 


\section{REFERENCES}

Aldred, D. N. (2013). Biological and Biomimetic Adhesives: Challenges and Opportunities. Cambridge: Royal Society of Chemistry.

Anders, S., Pyl, P. T., and Huber, W. (2015). HTSeq-a python framework to work with high-throughput sequencing data. Bioinformatics 31, 166-169. doi: 10.1093/bioinformatics/btu638

Dobin, A., Davis, C. A., Schlesinger, F., Drenkow, J., Zaleski, C., Jha, S., et al. (2013). STAR: ultrafast universal RNA-seq aligner. Bioinformatics 29, 15-21. doi: 10.1093/bioinformatics/bts635

Gorb, S. N. (2008). Biological attachment devices: exploring nature's diversity for biomimetics. Philos. Trans. A Math. Phys. Eng. Sci. 366, 1557-1574. doi: 10. 1098/rsta.2007.2172

Gruffydd, L. D. (1978). The byssus and byssus glands in Chlamys islandica and other scallops (Lamellibranchia). Zool. Scr. 7, 277-285. doi: 10.1111/j.14636409.1978.tb00611.x

Harrington, M. J., Masic, A., Holten-Andersen, N., Waite, J. H., and Fratzl, P. (2010). Iron-clad fibers: a metal-based biological strategy for hard flexible coatings. Science 328, 216-220. doi: 10.1126/science.1181044

Hayakawa, T., Yamashita, K., Tanzawa, K., Uchijima, E., and Iwata, K. (1992). Growth-promoting activity of tissue inhibitor of metalloproteinases-1 (TIMP1 ) for a wide range of cells. A possible new growth factor in serum. FEBS Lett. 298, 29-32. doi: 10.1016/0014-5793(92)80015-9

Huelsenbeck, J. P., Ronquist, F., Nielsen, R., and Bollback, J. P. (2001). Bayesian inference of phylogeny and its impact on evolutionary biology. Science 294, 2310-2314. doi: 10.1126/science.1065889

Iwamoto, M., Ueyama, D., and Kobayashi, R. (2014). The advantage of mucus for adhesive locomotion in gastropods. J. Theor. Biol. 353, 133-141. doi: 10.1016/j. jtbi.2014.02.024

Lee, B. P., Messersmith, P. B., Israelachvili, J. N., and Waite, J. H. (2011). Musselinspired adhesives and coatings. Annu. Rev. Mater. Res. 41, 99-132. doi: 10. 1146/annurev-matsci-062910-100429

Li, Y., Sun, X., Hu, X., Xun, X., Zhang, J., Guo, X., et al. (2017). Scallop genome reveals molecular adaptations to semi-sessile life and neurotoxins. Nat. Commun. 8:1721. doi: 10.1038/s41467-017-01927-0

Miao, Y., Zhang, L., Sun, Y., Jiao, W., Li, Y., Sun, J., et al. (2015). Integration of transcriptomic and proteomic approaches provides a core set of genes for understanding of scallop attachment. Mar. Biotechnol. (NY) 17, 523-532. doi: 10.1007/s10126-015-9635-y

Nagase, H. H., and Woessner, J. F. (1999). Matrix metalloproteinases. J. Biol. Chem. 274, 21491-21494. doi: 10.1074/jbc.274.31.21491

Naldrett, M. J., and Kaplan, D. L. (1997). Characterization of barnacle (Balanus eburneus and B-cenatus) adhesive proteins. Mar. Biol. 127, 629-635. doi: 10. $1007 / \mathrm{s} 002270050053$

Plumari, M., Gellera, C., and Taroni, F. (2010). Production of polyclonal antibodies against protein antigens purified by electroelution from SDS-polyacrylamide gel. Protoc. Exch. doi: 10.1038/nprot.2010.27

Qi, J. H., and Anand-Apte, B. (2015). Tissue inhibitor of metalloproteinase3 (TIMP3) promotes endothelial apoptosis via a caspase-independent mechanism. Apoptosis 20, 523-534. doi: 10.1007/s10495-014-1076-y

Qin, C. L., Pan, Q. D., Qi, Q., Fan, M. H., Sun, J. J., Li, N. N., et al. (2016). In-depth proteomic analysis of the byssus from marine mussel Mytilus coruscus. J. Proteomics 144, 87-98. doi: 10.1016/j.jprot.2016.06.014

Rao, S. K., Mathrubutham, M., Karteron, A., Sorensen, K., and Cohen, J. R. (1997). A versatile microassay for elastase using succinylated elastin. Anal. Biochem. 250, 222-227. doi: 10.1006/abio.1997.2223

Robinson, M. D., McCarthy, D. J., and Smyth, G. K. (2010). edgeR: a Bioconductor package for differential expression analysis of digital gene expression data. Bioinformatics 26, 139-140. doi: 10.1093/bioinformatics/btp616

Shevchenko, A., Tomas, H., Havlis, J., Olsen, J. V., and Mann, M. (2006). In-gel digestion for mass spectrometric characterization of proteins and proteomes. Nat. Protoc. 1, 2856-2860. doi: 10.1038/nprot.2006.468

Stetler-Stevenson, W. G., Bersch, N., and Golde, D. W. (1992). Tissue inhibitor of metalloproteinase-2 (TIMP-2) has erythroid-potentiating activity. FEBS Lett. 296, 231-234. doi: 10.1016/0014-5793(92)80386-U

Stohr, H., and Anand-Apte, B. (2012). A review and update on the molecular basis of pathogenesis of Sorsby fundus dystrophy. Adv. Exp. Med. Biol. 723, 261-267. doi: 10.1007/978-1-4614-0631-0_34
Sun, C., Lucas, J. M., and Waite, J. H. (2002). Collagen-binding matrix proteins from elastomeric extraorganismic byssal fibers. Biomacromolecules 3 , 1240-1248. doi: 10.1021/bm0255903

Tamura, K., Stecher, G., Peterson, D., Filipski, A., and Kumar, S. (2013). MEGA6: molecular evolutionary genetics analysis version 6.0. Mol. Biol. Evol. 30, 2725-2729. doi: 10.1093/molbev/mst197

Taylor, S. W., and Waite, J. H. (1997). "Marine adhesives: from molecular dissection to application," in Protein-Based Materials. Bioengineering of Materials, eds K. McGrath and D. Kaplan (Boston, MA: Birkhäuser), 217-248.

Toubarro, D., Gouveia, A., Ribeiro, R. M., Simoes, N., da Costa, G., Cordeiro, C., et al. (2016). Cloning, characterization, and expression levels of the nectin gene from the tube feet of the sea urchin Paracentrotus Lividus. Mar. Biotechnol. (NY) 18, 372-383. doi: 10.1007/s10126-016-9698-4

Tuuttila, A., Morgunova, E., Bergmann, U., Lindqvist, Y., Maskos, K., FernandezCatalan, C., et al. (1998). Three-dimensional structure of human tissue inhibitor of metalloproteinases-2 at 2.1 A resolution. J. Mol. Biol. 284, 1133-1140. doi: 10.1006/jmbi.1998.2223

Visse, R., and Nagase, H. (2003). Matrix metalloproteinases and tissue inhibitors of metalloproteinases: structure, function, and biochemistry. Circ. Res. 92, 827-839. doi: 10.1161/01.RES.0000070112.80711.3D

von Byern, J., Wani, R., Schwaha, T., Grunwald, I., and Cyran, N. (2012). Old and sticky-adhesive mechanisms in the living fossil Nautilus pompilius (Mollusca. Cephalopoda). Zoology (Jena) 115, 1-11. doi: 10.1016/j.zool.2011.08.002

Wang, C. S., and Stewart, R. J. (2013). Multipart copolyelectrolyte adhesive of the sandcastle worm, Phragmatopoma californica (Fewkes): catechol oxidase catalyzed curing through peptidyl-DOPA. Biomacromolecules 14, 1607-1617. doi: $10.1021 / \mathrm{bm} 400251 \mathrm{k}$

Wang, Q., Bao, Y., Huo, L., Gu, H., and Lin, Z. (2012). A novel tissue inhibitor of metalloproteinase in blood clam Tegillarca granosa: molecular cloning, tissue distribution and expression analysis. Fish Shellfish Immunol. 33, 645-651. doi: 10.1016/j.fsi.2012.06.021

Wang, S., Zhang, J., Jiao, W., Li, J., Xun, X., Sun, Y., et al. (2017). Scallop genome provides insights into evolution of bilaterian karyotype and development. Nat. Ecol. Evol. 1:120. doi: 10.1038/s41559-017-0120

Wilker, J. J. (2011). Biomaterials: redox and adhesion on the rocks. Nat. Chem. Biol. 7, 579-580. doi: 10.1038/nchembio.639

Willenbrock, F., Crabbe, T., Slocombe, P. M., Sutton, C. W., Docherty, A. J., Cockett, M. I., et al. (1993). The activity of the tissue inhibitors of metalloproteinases is regulated by C-terminal domain interactions: a kinetic analysis of the inhibition of gelatinase A. Biochemistry 32, 4330-4337. doi: $10.1021 / \mathrm{bi00067a023}$

$\mathrm{Xu}, \mathrm{W}$., and Faisal, M. (2008). Putative identification of expressed genes associated with attachment of the zebra mussel (Dreissena polymorpha). Biofouling 24, 157-161. doi: 10.1080/08927010801975345

Yang, B., Kang, D. G., Seo, J. H., Choi, Y. S., and Cha, H. J. (2013). A comparative study on the bulk adhesive strength of the recombinant mussel adhesive protein fp-3. Biofouling 29, 483-490. doi: 10.1080/08927014.2013.782541

Yang, J., Yan, R., Roy, A., Xu, D., Poisson, J., and Zhang, Y. (2015). The I-TASSER Suite: protein structure and function prediction. Nat. Methods 12, 7-8. doi: 10.1038/nmeth.3213

Zhang, G., He, L. S., Wong, Y. H., Xu, Y., Zhang, Y., and Qian, P. Y. (2015). Chemical component and proteomic study of the Amphibalanus (=Balanus) amphitrite shell. PLoS One 10:e0133866. doi: 10.1371/journal.pone.0133866

Zhao, H., Sun, C., Stewart, R. J., and Waite, J. H. (2005). Cement proteins of the tube-building polychaete Phragmatopoma californica. J. Biol. Chem. 280, 42938-42944. doi: 10.1074/jbc.M508457200

Conflict of Interest Statement: The authors declare that the research was conducted in the absence of any commercial or financial relationships that could be construed as a potential conflict of interest.

Copyright (C) 2018 Zhang, Dai, Wang, Miao, Xu, Liang, Dong, Bao, Wang, Lyu and Liu. This is an open-access article distributed under the terms of the Creative Commons Attribution License (CC BY). The use, distribution or reproduction in other forums is permitted, provided the original author(s) and the copyright owner are credited and that the original publication in this journal is cited, in accordance with accepted academic practice. No use, distribution or reproduction is permitted which does not comply with these terms. 